Análisis del tiempo de respuesta del laboratorio clínico al servicio de urgencia del Hospital del Niño Doctor José Renán Esquivel

\title{
ANÁLISIS DEL TIEMPO DE RESPUESTA DEL LABORATORIO CLÍNICO AL SERVICIO DE URGENCIA DEL HOSPITAL DEL NIÑO DOCTOR JOSÉ RENÁN ESQUIVEL DURANTE EL SEGUNDO TRIMESTRE DE 2016
}

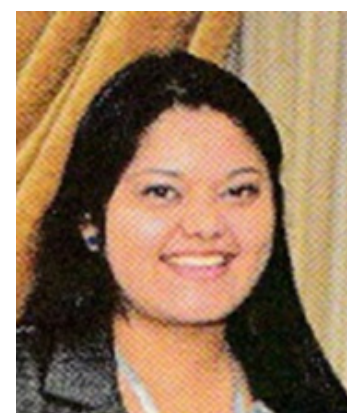

\author{
Yaremi del Carmen Juárez Moore \\ Hospital del Niño Doctor José Renán Esquivel. Panamá \\ Yaremi.juarez@gmail.com
}

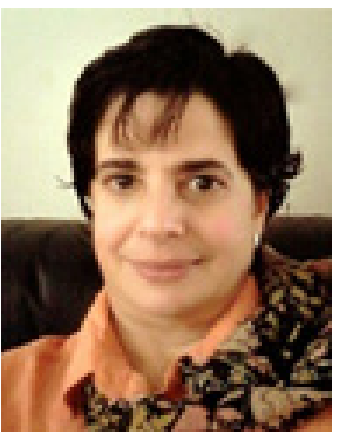

\section{Celia Llanusa Ruiz \\ Universidad Santander- USANTANDER. Panamá \\ Celia.1lanusa@usantander.edu.pa}

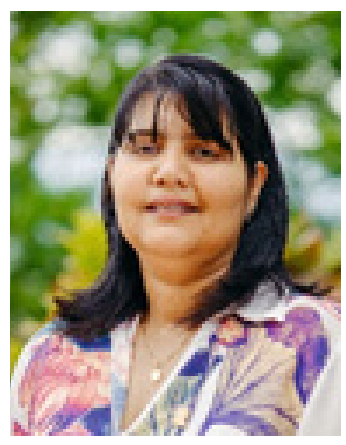

\section{Johana Gutiérrez Zehr \\ Universidad de Santander- UDES. Colombia \\ Johana.gutierrez@udes.edu.co}

\section{RESUMEN}

El objetivo del presente estudio fue evaluar los tiempos de respuesta del laboratorio clínico para los pacientes del servicio de urgencia del Hospital del Niño Doctor José Renán Esquivel, que acudieron a consulta en el segundo trimestre 2016. En cuanto al diseño se aplicó el Método descriptivo-restrospectivo. Universos compuestos por pacientes que acudieron a urgencias del hospital y que requierieron análisis clínicos entre los meses de abril a junio de 2016: 
Análisis del tiempo de respuesta del laboratorio clínico al servicio de urgencia del Hospital del Niño Doctor José Renán Esquivel

Universo \#1: 5.000 pacientes con análisis clínico hematológico. 357muestras analizadas. Universo \#2: 3.023 pacientes con análisis clínico de química general. 342 muestras analizadas. Universo \#3: 1.689 pacientes con análisis clínico de urinálisis. 313 muestras analizadas. Universo \#4: 420 pacientes con análisis clínico de parasitología. 201 muestras analizadas.

En cuanto a los materiales y métodos se generó la información mediante la revisión y análisis documental de las solicitudes médicas ingresadas al sistema de información de laboratorio clínico MODULAB. Como conclusión del estudio se identificó que la sección que posee el mayor porcentaje de informes de laboratorio clínico fuera de tiempo es hematología general con un $40.62 \%$ seguida de la sección de química general con un $25.14 \%$. Todas las secciones cumplen con tiempos de respuestas globales menores a 1:00:00 hora, pero existen tendencias de tiempos de respuesta prolongados en los horarios matutinos y vespertinos.

Palabras clave: tiempo de respuesta, laboratorio clínico, urgencia, calidad.

\title{
ANALYSIS OF THE RESPONSE TIME OF THE CLINICAL LABORATORY TO THE EMERGENCY SERVICE OF THE NIÑO DOCTOR HOSPITAL JOSÉ RENÁN ESQUIVEL DURING THE SECOND QUARTER OF 2016
}

\begin{abstract}
The objective of the present study was to evaluate the response times of the clinical laboratory for patients of the emergency service of the Doctor José Renán Esquivel Children's Hospital, who attended the consultation in the second quarter of 2016. As for the design, the Descriptive Method was applied retrospective Universes composed of patients who came to the hospital's emergency room and who required clinical analysis between the months of April to June 2016: Universe \# 1: 5,000 patients with clinical hematological analysis. 357 samples analyzed. Universe \# 2: 3,023 patients with clinical analysis of general chemistry. 342 samples analyzed. Universe \# 3: 1,689 patients with clinical analysis of urinalysis. 313 samples analyzed. Universe \# 4: 420 patients with clinical analysis of parasitology. 201 samples analyzed.
\end{abstract}

In terms of materials and methods, the information was generated through the review and documentary analysis of the medical applications entered into the MODULAB clinical laboratory information system. As a conclusion of the study, it was identified that the section with the highest percentage of clinical laboratory reports out of time is general hematology with $40.62 \%$ followed by the general chemistry section with $25.14 \%$. All sections comply with global response times 
Análisis del tiempo de respuesta del laboratorio clínico al servicio de urgencia del Hospital del Niño Doctor José Renán Esquivel durante el segundo trimestre de 2016

Yaremi del Carmen Juárez Moore - Celia Llanusa Ruiz - Johana Gutiérrez Zehr (42:56)

less than 1:00:00 hour, but there are trends in extended response times in morning and evening hours.

Key words: response time, clinical laboratory, urgency, quality

\section{INTRODUCCIÓN}

La demanda de los servicios de urgencia pediátricos aumenta cada año, y el rol del laboratorio clínico tiene un papel clave en los tiempos de atención a los pacientes que acuden a dichas instalaciones. Según estimaciones oficiales de la República de Panamá al $1^{\circ}$ de junio de 2014, la población fue de 3.913,275 y la población menor de 15 años de 1.087.57, representando el 27,8\% de la población total, siendo su crecimiento con respecto al año anterior de 3,1\%. El Hospital del Niño Doctor José Renán Esquivel es una institución del tercer nivel de atención y cuarto nivel de complejidad, cuya misión es ofrecer servicios de atención pediátrica, con calidad y humanismo. Haciendo énfasis en la solución integral de problemas de alta complejidad. Dentro del boletín estadístico que presentó el Hospital en el año 2014, se reportó que la atención especializada aumentó en un $7.6 \%$ con respecto al año anterior con énfasis en la atención de urgencia, dado que de los 199.616 pacientes que consultaron, el 51,3\% fueron atendidos en este servicio. Hospital del Niño Doctor José Renán Esquivel (2014)

El Hospital en su servicio de urgencia no cuenta con la figura de un laboratorio de urgencia que se encuentre físicamente próximo y que sea parte integral y exclusiva de dicho servicio, sino que se utiliza el laboratorio clínico y especializado tanto para los pacientes que acuden a urgencia como para aquellos que acuden por consulta externa y pacientes hospitalizados. En promedio diariamente en la institución se atienden 280 consultas de pediatría general y de urgencia, por lo que brindar un servicio eficiente, es un indicador de calidad en la atención a los pacientes, el cual garantiza la satisfacción de los usuarios que acuden al centro hospitalario. Es por ello, que la investigación buscó evaluar los tiempos de respuestas del laboratorio clínico para el servicio de urgencia del hospital, durante el segundo trimestre 2016.

Para el año 2016 el servicio de laboratorio clínico contaba con un total de 35 tecnólogos médicos, 10 técnicos asistentes de laboratorio clínico, dos auxiliares de laboratorio clínico y nueve secretarias. El horario de atención es de lunes a viernes en donde se establecen cuatro turnos así: $7 \mathrm{am}-3 \mathrm{pm}, 3 \mathrm{pm}-9 \mathrm{pm}, 7 \mathrm{pm}$ - 1am, 1am - 7am y el número de personal varía dependiendo de la necesidad de servicio. Los fines de semana se dividen de la siguiente manera: 7am - 1pm (cinco tecnólogos médicos, un técnico asistente, un auxiliar de laboratorio, un personal de secretaria), $1 \mathrm{pm}-7 \mathrm{pm}$ (dos tecnólogos médicos, un técnico asistente, un personal de secretaria), 7pm lam (dos tecnólogos médicos, un técnico asistente, un personal de secretaria) y 1am - 7am (dos 
Análisis del tiempo de respuesta del laboratorio clínico al servicio de urgencia del Hospital del Niño Doctor José Renán Esquivel durante el segundo trimestre de 2016 Yaremi del Carmen Juárez Moore - Celia Llanusa Ruiz - Johana Gutiérrez Zehr (42:56)

tecnólogos médicos, un técnico asistente, un personal de secretaria).

En el Hospital del Niño, el 76,6\% de los pacientes que acuden al servicio de urgencia provienen de la provincia de Panamá y el 23,4\% de otras provincias, de este porcentaje de pacientes que provienen de las otras provincias, el 35,3\% acuden a la atención de urgencia y el $65,7 \%$ a la consulta especializada, lo que indica que esta institución continúa siendo un hospital de referencia, a nivel nacional. Las causas más frecuentes de consulta en el servicio de urgencia son las enfermedades respiratorias con un $25,1 \%$ y las diarreicas $10,5 \%$. Estas causas se mantienen a través de los años por la climatología la cual ha permanecido dentro de los parámetros de estación seca y lluviosa. Hospital del Niño Doctor José Renán Esquivel (2014).

El manual de procedimiento de recepción del laboratorio clínico contempla que los exámenes solicitados por parte del servicio de urgencia deben ser de prioridad y procesados en un tiempo no mayor de una hora (1:00:00), es decir, que si el personal del laboratorio clínico no cumple con los tiempos de respuestas descritos en el manual, el tiempo de atención al paciente puede verse afectado, y por ende la calidad de atención que se brinda en el servicio de urgencia del hospital, dado que su efecto será la demora en la toma de decisiones por parte del personal clínico.

La función del servicio de laboratorio clínico en urgencias es ofrecer resultados precisos, confiables, y de forma oportuna para lograr un verdadero apoyo diagnóstico. Los resultados ayudan a confirmar una impresión clínica, establecer un diagnóstico o descartarlo, realizar una exploración selectiva, obtener una información pronostica de una enfermedad, conocer la respuesta terapéutica y precisar factores de riesgo. El tiempo de espera del paciente en los servicios de urgencia constituye una constante preocupación, pues usualmente tiene que realizar períodos de espera variables y en ocasiones no es debidamente informado, esto causa molestias que podrían ser evitadas. Por esta razón se le debe hacer saber al paciente que su espera en emergencias puede deberse a que hay otros pacientes con cuadros más graves e incluso con peligro de muerte y y que tienen que ser vistos primero. Otras situaciones concretas pueden demorar el proceso de los servicios de urgencia como las epidemias, la necesidad de consultar otros especialistas o la espera de exámenes complementarios como los estudios de laboratorio y/o rayos X.

\section{FUNDAMENTACIÓN}

Los pacientes que acuden al servicio de urgencia de una institución de salud llegan con estados críticos, que requieren de una atención eficiente, pero sin dejar de lado los estándares de calidad necesarios para un correcto diagnóstico y tratamiento. El Laboratorio clínico es considerado un servicio complementario para el diagnóstico y tratamiento del paciente por parte del personal clínico, por ende juegan un papel sumamente importante el análisis correcto y 
Análisis del tiempo de respuesta del laboratorio clínico al servicio de urgencia del Hospital del Niño Doctor José Renán Esquivel durante el segundo trimestre de 2016 Yaremi del Carmen Juárez Moore - Celia Llanusa Ruiz - Johana Gutiérrez Zehr (42:56)

los tiempos para emisión de resultados, elementos clave en la toma de decisiones médicas. El Tiempo de Respuesta (TR) se define en esta investigación como el tiempo que transcurre desde el momento en que se genera la petición en el sistema de información para la realización de los exámenes hasta la emisión del informe de laboratorio y su envío vía web al médico de urgencia. El TR es considerado por los programas de garantía de calidad como un indicador de la eficacia de los laboratorios, siendo imprescindible su medición sistemática y análisis, para garantizar la calidad extra analítica. A pesar de los avances en la tecnología de análisis, sistemas de transporte y la informatización, muchos laboratorios han tenido dificultades para mejorar sus TR.

En algunas situaciones agudas, pueden necesitarse los resultados en minutos luego de solicitados y en otros casos, un TR de una hora o dos puede ser aceptable. Los médicos utilizan los resultados del laboratorio para confirmar o modificar su impresión clínica y ellos frecuentemente deben tomar decisiones en términos de minutos. Es por esto que los plazos de entrega de resultados deben ser vigilados, registrados y revisados para tomar acciones correctivas en caso de identificar problemas. Shmukleman, (2010).

Como antecedentes para esta investigación se tomaron en cuenta estudios internacionales que evalúan la satisfacción de los usuarios en los servicios de emergencias, lo cual se ha relacionado en gran medida con los tiempos de respuesta de los exámenes clínicos. En los Estados Unidos se han llevado a cabo investigaciones sobre el uso de los análisis de laboratorio por parte del personal médico y se ha concluido que en muchos casos, los análisis solicitados no eran relevantes para el manejo del paciente. Igualmente se ha realizado evaluación del papel del personal de laboratorio en función de la práctica médica moderna. Evidenciando la dependencia del diagnóstico clínico a partir de los resultados de laboratorio. Cascante (2007).

En el año 2001, se publicaron los resultados del estudio en un Laboratorio Central del Complejo Hospitalario de la Rioja en España, donde se comparaban los tiempos de respuesta en dos períodos diferentes, concluyéndose que el tiempo medio intra-laboratorio se incrementó en un 44\% desde el estudio de 1998, mientras que el tiempo medio total desde que el médico realiza la solicitud hasta que recibe los resultados, disminuyó en un 34\%. Cascante (2007).

Varios estudios han señalado que los tiempos promedio de duración de asistencia médica y estancia están directamente relacionados con la gravedad del paciente. En la actualidad las organizaciones exitosas de cualquier tipo ponen especial énfasis en la reducción de sus tiempos de espera, en Panamá muchos laboratorios clínicos no consideran como uno de los principales focos de atención la oportunidad en los tiempos de entrega de resultados, en contraste con esta realidad, los médicos juzgan la eficiencia de un laboratorio clínico con base en la rapidez con que reportan sus resultados. Pérez J, f. Limón f. (2008). 
Análisis del tiempo de respuesta del laboratorio clínico al servicio de urgencia del Hospital del Niño Doctor José Renán Esquivel durante el segundo trimestre de 2016

Yaremi del Carmen Juárez Moore - Celia Llanusa Ruiz - Johana Gutiérrez Zehr (42:56)

A nivel internacional se han desarrollado estudios de satisfacción de usuarios en los sistemas de salud. Por ejemplo, en el año 2011 se realizó un estudio en Costa Rica cuyo objetivo general era el de analizar la satisfacción de usuario del laboratorio clínico; el estudio cuantitativo aplicó encuestas a 344 pacientes que acudieron al centro de atención de Cariari y entre sus resultados se destaca la insatisfacción en el tiempo de respuesta del laboratorio clínico, especialmente la espera de los pacientes para recibir los resultados clínicos. Moraga M, González C (2011).

En Valencia, España se realizó un estudio en el cual se comparaban los tiempos de respuestas de ocho laboratorios clínicos, el cual arrojó resultados con tiempos de respuestas prolongados y cuyas recomendaciones fueron las de continuar monitoreando los tiempos de respuestas con miras a mejoras, así como el rediseño de procesos y darle continuidad al benchmarking. Salinas, M., López-Garrigós, M., Yago, M., Ortuño, M., Díaz, J., Marcaida, G., Uris, J., (2011).

Los aspectos normativos de calidad contemplan la norma ISO 9001:2015, la cual es internacional, genérica, y establece los requisitos que debe cumplir el sistema de gestión de la calidad de una organización, propone una metodología encaminada a mejorar los aspectos organizativos mediante la mejora de los procesos, enfocándolos al paciente y con participación activa de los profesionales.

Esta certificación de calidad está orientada para aumentar la satisfacción de los pacientes, mediante una asistencia de mayor calidad, así como por mejorar la labor de los profesionales del área implicada, ya que el sistema permite optimizar los conocimientos y los medios técnicos disponibles, planificando el trabajo de forma más eficaz y eficiente.

Un sistema de gestión de calidad basado en la Norma 15189:2012 y 9001:2015 podría significar reducir de raíz las fallas que resultan en No Conformidades y que generan procesos duplicados (White, 2002), al igual que establece que la monitorización debe ser planeada, lo cual incluye establecer objetivos, metodologías, interpretaciones, limitaciones, planes de acción y duración de la medición, además de ser revisados periódicamente para asegurar una continuidad apropiada; pero la norma también explicita que finalmente la institución es quien debe establecer los tiempos de respuesta para cada uno de los exámenes que reflejen las necesidades clínicas y que se deberá evaluar periódicamente si se está o no se está cumpliendo con los plazos de entrega establecidos. International Organization for Standardization (2012).

Cascante (2007) pone de manifiesto la importancia del aseguramiento de la calidad en el laboratorio clínico, indicando que debería ir más allá de esta norma e incluir, también, en los objetivos de calidad de sus resultados finales, su contribución a la mejora de la calidad del nivel médico y asistencial de su entorno y así mismo del uso de los recursos humanos y materiales puestos a su disposición, adicionalmente declara que se debe evaluar la calidad de la asistencia ofrecida 
Análisis del tiempo de respuesta del laboratorio clínico al servicio de urgencia del Hospital del Niño Doctor José Renán Esquivel durante el segundo trimestre de 2016

Yaremi del Carmen Juárez Moore - Celia Llanusa Ruiz - Johana Gutiérrez Zehr (42:56)

en los servicios de urgencias y que es necesario utilizar un grupo de herramientas que permitan considerar los aspectos más importantes, al igual que su monitorización. Una propuesta para conocer la calidad asistencial, es la evaluación por indicadores, la cual ha mostrado ser un instrumento útil para este fin. Manifiesta que las diferencias entre los servicios de urgencia radican en términos de ubicación, población de usuarios meta, recursos humanos y de infraestructura disponible, tamaño y capacidad del hospital, número de especialistas y mecanismos de referencia y contra referencia.

Según la Resolución 1 del 31 de mayo de 1984 "Por la cual se establece las normas para apertura y operación de los laboratorios de análisis clínicos” en Panamá, en el Capítulo III Artículo 21 de la clasificación de los laboratorios de análisis clínicos, los laboratorios serán clasificados, de acuerdo con los análisis que efectúan en sus instalaciones, en laboratorio de análisis clínicos Clase 1 y 2. Laboratorio clase 1: se describe como aquel autorizado para efectuar pruebas básicas de: hematología general, química general, serología general, urinálisis y parasitología general. Laboratorio clase 2: se describe como autorizado para efectuar pruebas generales y especializadas en hematología, inmunoserología, química especial, microbiología (bacteriología y micología), hormonas, toxicología, inmunología, genética y otras disciplinarias de las ciencias de laboratorio que requieran de equipos más sofisticados y recursos humanos debidamente capacitados $\mathrm{y}$ cónsonos con la especialidad que se efectúe en el laboratorio. De acuerdo con lo descrito en la Resolución, el laboratorio clínico del Hospital del Niño Doctor José Renán Esquivel está dentro de la clasificación de laboratorio clase 2. Dentro de estos laboratorios Clase 2, cada uno de los procesos pre analíticos, analíticos y post analíticos, deben ser monitoreados, para garantizar la confiabilidad y calidad de los resultados o informes que se emiten en el laboratorio clínico, y los cuales son de apoyo diagnóstico y de gran importancia en la toma de decisiones por parte del personal clínico.

Según García L, Boned J (2012), el proceso del análisis clínico se divide en tres fases a saber:

Fase Pre analítica: lo más habitual es registrar todas las incidencias que se pueden presentar en esta fase: desde la calidad de la cumplimentación de la solicitud, la preparación del paciente, problemas en la extracción e identificación de los especímenes, calidad de las muestras, etc. (Todos los estudios realizados sobre errores en la práctica de los laboratorios clínicos coinciden en que la mayoría de ellos suceden en las fases extra analíticas y más concretamente en la pre analítica, siendo además en esta última, los más críticos).

Fase Analítica: es la más establecida y la primera que se implantó en los laboratorios clínicos. (A veces se tiende a menospreciar por considerar que está "superada", debido a la mejora de los sistemas analíticos que actualmente se emplean en los laboratorios. Sin embargo, la realidad no es esa, por lo que debemos seguir muy atentos al control de la calidad analítica).

Fase Post analítica: también en este caso, lo más frecuente es registrar todas las incidencias que se puedan generar. (El desarrollo de programas y conexiones informáticas ha logrado mejorar los aspectos relacionado a esta fase. 
Análisis del tiempo de respuesta del laboratorio clínico al servicio de urgencia del Hospital del Niño Doctor José Renán Esquivel durante el segundo trimestre de 2016 Yaremi del Carmen Juárez Moore - Celia Llanusa Ruiz - Johana Gutiérrez Zehr (42:56)

Sin embargo, quedan importantes áreas de mejora como la explotación de datos, para transformarlos en información útil, o el desarrollo e implantación de sistemas expertos).

En referencia al sistema Informático clínico utilizado para este estudio consistió en un Laboratorio de Información Integrada (LIS) llamado MODULAB, basado en las últimas tecnologías disponibles. Este sistema permite brindar distintas soluciones para la generación de los informes del laboratorio clínico, cuenta con una integración completa involucrando desde el proceso de flujo de trabajo: de administración y registro de pacientes, a la flebotomía, la recepción de la muestra, la solicitud electrónica, que abarca el proceso de análisis, validación y presentación de informes, estadísticas, gestión de inventarios y más. Este sistema permite monitorear los tiempos de respuestas de los laboratorios a partir del momento en que son ingresados al sistema y puede configurarse para brindar al servicio de urgencia prioridad en sus resultados.

La existencia de los servicios de urgencia en los centros hospitalarios ha venido a representar un aspecto importante en el tratamiento de los pacientes, estos servicios tienen como principal objetivo dar una atención pronta y oportuna como sea posible y con un máximo de calidad, $\mathrm{y}$, de este modo, agilizar las acciones médicas para poder garantizarle al paciente su bienestar. Los tiempos de espera de los resultados del laboratorio en muchas instituciones de salud causan insatisfacción, cuando éstos son mayores que los que el paciente considera apropiados o porque no ha sido debidamente informado del tiempo que demorará el resultado.

\section{METODOLOGÍA}

Esta investigación fue de tipo descriptivo, analítico de corte retrospectivo, que incluyó consulta bibliográfica, revisión de informes de laboratorios clínicos de hematología, química general, urinálisis y parasitología, en el segundo trimestre de 2016. Con el fin de identificar los TR y las causas de posibles retrasos en los mismos.

Respecto a la población participante, fueron todos los pacientes que acudieron al servicio de urgencia en el trimestre seleccionado y la muestra, estuvo representada en cuatro universos. Las muestras fueron calculadas con base al programa de Epi Info Stat Cal (Centers for Disease Control and Prevention, 2013) con 2 desviaciones estándar y un 95\% de certeza.

Universo \#1: 5.000 pacientes con análisis clínico hematológico.

La muestra analizada fue de 357 solicitudes de exámenes hematológicos

Universo \#2: 3.023 pacientes con análisis clínico de química general.

La muestra analizada fue de 342 solicitudes de exámenes de química general.

Universo \#3: 1.689 pacientes con análisis clínico de urinálisis.

La muestra analizada fue de 313 solicitudes de exámenes de urinálisis. 
Análisis del tiempo de respuesta del laboratorio clínico al servicio de urgencia del Hospital del Niño Doctor José Renán Esquivel durante el segundo trimestre de 2016 Yaremi del Carmen Juárez Moore - Celia Llanusa Ruiz - Johana Gutiérrez Zehr (42:56)

Universo \#4: 420 pacientes con análisis clínico de parasitología.

La muestra analizada fue de 201 peticiones de exámenes de parasitología.

Cabe aclarar que a pesar de que la sección de urinálisis es una y trabaja el mismo tecnólogo médico, fueron analizadas por separado debido al proceso en la recolección de la muestra, ya que en general no se ingresan a la vez las solicitudes, generándose informes separados e independientes.

La fuente de información fueron las peticiones que se generaron al ingresar las solicitudes médicas al sistema, las cuales aportaron los datos para el análisis de los tiempos de respuestas. Del sistema MODULAB se seleccionaron muestras al azar y se procedió a su registro en un formulario creado para tal fin.

En referencia a la Técnica e instrumentos para la recolección de datos, se tuvo en cuenta la revisión y análisis documental de los informes de laboratorio en el sistema MODULAB.

Como criterios de inclusión se tomaron en cuenta los siguientes aspectos: Solicitudes médicas de pacientes procedentes del servicio de urgencia para pruebas de hematología, química general, urinálisis o parasitología cada sección de forma individual, que fueron recibidas, ingresadas al sistema de información del laboratorio clínico y contaban con informes completos y enviados.

Los datos fueron procesados a través del siguiente procedimiento: ingreso al sistema MODULAB con un usuario y contraseña, ingreso a pestaña de analítica, ingreso a Gestión de Peticiones, tabulación de datos para el filtro de la siguiente manera: fechas que se deseaban revisar, servicio de urgencia, sección (elegir hematología, urinálisis y parasitología, química general), se indicaba el estado de la petición "todas" y finalmente se registraban los datos.

Respecto a la conducción del estudio el sitio de investigación para el desarrollo de esta investigación fue el Laboratorio Clínico del Hospital del Niño Doctor José Renán Esquivel de Ciudad de Panamá y en cuanto a los aspectos éticos, la información del estudio se manejó con el consentimiento previo de la institución y la propuesta fue remitida al comité de Bioética en Investigación de la misma. Las actividades en este estudio se realizaron de manera responsable, con ética profesional, confidencialidad y con respeto a los valores y principios de la comunidad y de cada universidad participante.

No se utilizan datos demográficos de los pacientes, por lo que su información fue manejada con estricta confidencialidad; se considera investigación de bajo riesgo, debido a que solo se manejaron y calcularon datos. 
Análisis del tiempo de respuesta del laboratorio clínico al servicio de urgencia del Hospital del Niño Doctor José Renán Esquivel

\section{RESULTADOS}

Los resultados fueron derivados a partir de la revisión de 1.213 informes de laboratorio clínico del Hospital del Niño Dr. José Renán Esquivel de la ciudad de Panamá, de los cuales 357 fueron de hematología, 342 química general, 313 de urinálisis y 201 de parasitología, en el periodo de los meses de abril, mayo y junio de 2016. En la Tabla 1 se aprecia los tiempos de respuesta por cada sección analizada. La sección de parasitología tuvo menor tiempo promedio de atención comparada con las demás secciones (38 minutos), con un $85 \%$ de informes revisados dentro del tiempo establecido (172 pruebas) y con un $15 \%$ de pruebas fuera de tiempo (29 pruebas).

Tabla 1. Estadísticas de los tiempos de respuestas por sección analizada en el segundo trimestre del año 2016.

\begin{tabular}{|c|c|c|c|c|c|c|}
\hline \multirow[b]{2}{*}{ Sección } & \multirow[b]{2}{*}{$\mathbf{n}$} & \multirow[b]{2}{*}{ Promedio } & \multirow[b]{2}{*}{\begin{tabular}{l}
\multicolumn{1}{c}{$\mathrm{N}^{\circ}$} \\
de \\
pruebas \\
fuera \\
de \\
tiempo
\end{tabular}} & \multicolumn{3}{|c|}{$\mathbf{N}^{\circ}$} \\
\hline & & & & \begin{tabular}{l}
\multicolumn{1}{c}{$\%$ de } \\
pruebas \\
fuera de \\
tiempo
\end{tabular} & $\begin{array}{l}\text { de } \\
\text { pruebas } \\
\text { dentro } \\
\text { de } \\
\text { tiempo }\end{array}$ & \begin{tabular}{l}
\multicolumn{1}{c}{$\%$} \\
pruebas \\
dentro de \\
tiempo
\end{tabular} \\
\hline Hematología & 357 & $0: 59 \mathrm{~min}$ & 145 & $40.62 \%$ & 212 & $59.38 \%$ \\
\hline Q. General & 342 & $0: 52 \mathrm{~min}$ & 85 & $25.14 \%$ & 257 & $74.85 \%$ \\
\hline Urinálisis & 313 & $0: 41 \mathrm{~min}$ & 48 & $15.34 \%$ & 265 & $84.66 \%$ \\
\hline Parasitología & 201 & $0: 38 \mathrm{~min}$ & 29 & $14.43 \%$ & 172 & $85.57 \%$ \\
\hline
\end{tabular}

Fuente: Informes de laboratorio clínico dentro del sistema informático MODULAB, del laboratorio clínico Hospital del Niño Dr. José Renán Esquivel.

La Tabla 2 muestra los promedios de los tiempos de respuesta de la sección de hematología, en cada turno, donde se puede observar que el turno de $3 \mathrm{pm}-9 \mathrm{pm}$ de los días de semana y el $7 \mathrm{am}$ - 3pm también de los días de semana tiene un promedio de tiempo de respuesta mayor a 1:00:00 hora. El promedio de tiempo de respuesta del turno de 7am-3pm es de 1:10:44 y el del 3pm-9pm es de 1:01:12, a pesar de que en ambos turnos el personal disponible está a cargo solo de esa sección, relacionándose no con el número de personal disponible ni las secciones a cargo, pero si con el número elevado de solicitudes de urgencias que ingresan en esos horarios.

En los turnos mencionados anteriormente se pudo observar que existen tendencias en ciertos horarios. En el turno de 7am-3pm día de semana, las horas en donde se observan la mayoría de los tiempos de respuesta prolongados son de 6:30 am-9:30 am, se considera que la causa es que en ese horario el personal suele realizar los procesos de mantenimiento y control de calidad de los equipos, y van integrándose de manera escalonada hasta las 9 am que es cuando están todas las unidades disponibles en el turno de 8 horas. También suele existir un máximo de dos tecnólogos médicos para realizar todo lo anteriormente mencionado y además atender las solicitudes tanto de 
urgencia como de consultas externas y hospitalizadas en dichos horarios. En el horario de horario de 3pm a 9pm día de semana, la tendencia se muestra en las horas de 5:00 pm a 7:00 pm.

El turno de $3 \mathrm{pm}$ a 9pm los días de semana tiene un porcentaje de peticiones de urgencias fuera de tiempo de $26,90 \%$ y el $7 \mathrm{am}$ - 3pm en días de semana de 37,2\%, siendo los turnos más afectados, y a pesar de que el turno de $7 \mathrm{am}$ a $3 \mathrm{pm}$ tiene la mayor cantidad de personas involucradas en el proceso y sólo una sección a cargo, posee el mayor porcentaje con tiempo de respuestas mayores a 1:00:00 hora.

Tabla 2. Tiempos de respuestas promedio de la sección de Hematología por Turno

\begin{tabular}{lll}
\hline Turno & $\mathbf{n}=\mathbf{3 5 7}$ & Promedio \\
\hline lam - 7am (Día de semana) & 22 & $0: 49$ \\
\hline 7am - 3pm (Día de semana) & 103 & $1: 10: 44$ \\
\hline 3pm - 9pm (Día de semana) & 83 & $1: 01: 12$ \\
\hline 7pm - lam (Día de semana) & 38 & $0: 50: 38$ \\
\hline lam - 7am (fin de semana) & 17 & $0: 43$ \\
\hline 7am - lpm (fin de semana) & 29 & $0: 53: 29$ \\
\hline lpm - 7pm (fin de semana) & 32 & $0: 48: 15$ \\
\hline 7pm - lam (fin de semana) & 33 & $0: 58: 11$ \\
\hline
\end{tabular}

Fuente: Informes de laboratorio clínico dentro del sistema informático MODULAB, del laboratorio clínico Hospital del Niño Dr. José Renán Esquivel.

Los turnos analizados en Química general cumplen con el tiempo de respuesta máximo permitido en el manual de procedimiento (60 minutos) según la Tabla 3. Los días de semana en la sección de química general, se observa exactamente igual que en hematología general en los horarios de $7 \mathrm{am}-3 \mathrm{pm}$ y de $3 \mathrm{pm}-9 \mathrm{pm}$, existen tendencias en los horarios matutinos de $7 \mathrm{am}-$ 9:30 am, coincidiendo con los procesos de mantenimiento y control de los equipos, y en el turno vespertino de 4:30 pm-6:00 pm. El porcentaje de informes de laboratorio fuera de tiempo más elevado se concentra en el turno de $7 \mathrm{am}-3 \mathrm{pm}$ los días de semana con un $37.21 \%$, seguido del turno de 3 pm-9pm con un $29.07 \%$.

Tabla 3. Tiempos de respuestas promedio de la sección de Química General por turno.

\begin{tabular}{lll}
\hline Turno & $\mathbf{n = 3 4 2}$ & Promedio \\
\hline lam - 7am (día de semana) & 29 & $0: 48: 02$ \\
lam - 7am (fin de semana) & 8 & $0: 49: 07$ \\
3pm - 9pm (día de semana) & 77 & $0: 53: 57$ \\
\hline 7pm - lam (día de semana) & 38 & $0: 46: 05$ \\
7pm - lam (fin de semana) & 25 & $0: 44 ; 38$ \\
\hline 7am - lpm (fin de semana) & 27 & $0: 58: 38$ \\
\hline 7am - 3pm (día de semana) & 99 & $0: 57: 48$ \\
\hline lpm - 7pm (fin de semana) & 39 & $0: 43: 11$ \\
\hline
\end{tabular}

Fuente: Informes de laboratorio clínico dentro del sistema informático MODULAB, del laboratorio clínico Hospital del Niño Dr. José Renán Esquivel 
La sección de urinálisis y parasitología a pesar de ser una sola sección, se estudió de manera separada, pero es el mismo personal en todos los turnos. La sección de urinálisis en los diferentes turnos, tiene promedios de tiempo de repuesta menores a 1:00:00 hora, es decir que son aceptables de acuerdo a la Tabla 4. Los informes de laboratorios de la sección de urinálisis fuera de tiempo corresponden a 48 , de los cuales el $25 \%$ corresponde al turno de $3 \mathrm{pm}$ - 9pm de los días de semana y el $27,1 \%$ al turno de $7 \mathrm{am}-3 \mathrm{pm}$ en días de semana siendo estos los dos turnos más afectados, y este último en mención el único turno que mantiene un solo personal disponible para la realización de los procesos.

Tabla 4. Tiempos de respuestas promedio de la sección de Urianálisis por turno

\begin{tabular}{|lll}
\hline TURNO & $\mathbf{n}=\mathbf{3 1 3}$ & Promedio \\
\hline lam - 7am (día de semana) & 32 & $0: 38: 00$ \\
\hline lam - 7am (fin de semana) & 8 & $0: 33: 00$ \\
\hline 3pm - 9pm (día de semana) & 69 & $0: 42: 39$ \\
\hline 7pm - lam (día de semana) & 34 & $0: 40: 11$ \\
\hline 7pm - lam (fin de semana) & 20 & $0: 47: 51$ \\
\hline 7am - lpm (fin de semana) & 23 & $0: 46: 21$ \\
\hline 7am - 3pm (día de semana) & 104 & $0: 42: 09$ \\
\hline lpm - 7pm (fin de semana) & 23 & $0: 40: 00$ \\
\hline
\end{tabular}

Fuente: Informes de laboratorio clínico dentro del sistema informático MODULAB, del laboratorio clínico Hospital del Niño Dr. José Renán Esquivel.

El promedio de los tiempos de respuestas en la sección de parasitología por turno, se evidencian que son menores a 1:00:00 hora, es decir, aceptable dentro del manual de procedimientos. Los turnos con porcentajes de informes de laboratorio con mayor afectación son los turnos del $7 \mathrm{am}-3 \mathrm{pm}$ los días de semana y el 3pm - 9pm los días de semana con un 41,38\% y 24,14\% respectivamente, pudiéndose observar que en el 7am - 3pm existe también un solo personal a cargo de la sección. La sección de urinálisis con un tiempo de respuesta promedio de 0:41:00 minutos y parasitología 0:38:00 minutos, también mostraron tendencia en el turno de 7am a 3pm en horario de 7:00 am a 9:30 am, en este punto hay que considerar la programación del personal ya que no existe uno exclusivo en la sección en ese lapso de tiempo (Véase Tabla 5).

Tabla 5. Tiempos de respuestas promedio de la sección de Parasitología por turno

\begin{tabular}{lll}
\hline TURNO & $\mathbf{n}=\mathbf{2 0 1}$ & Promedio \\
\hline lam - 7am (día de semana) & 8 & $0: 41: 00$ \\
\hline lam - 7am (fin de semana) & 8 & $0: 43: 00$ \\
\hline 3pm - 9pm (día de semana) & 54 & $0: 34: 40$ \\
\hline 7pm - lam (día de semana) & 16 & $0: 47: 07$ \\
\hline 7pm - lam (fin de semana) & 7 & $0: 54: 12$ \\
7am - lpm (fin de semana) & 15 & $0: 34: 36$ \\
\hline 7am - 3pm (día de semana) & 76 & $0: 39: 30$ \\
\hline lpm - 7pm (fin de semana) & 17 & $0: 30: 15$ \\
\hline
\end{tabular}

Fuente: Informes de laboratorio clínico dentro del sistema informático MODULAB, del laboratorio clínico Hospital del Niño Dr. José Renán Esquivel 
Análisis del tiempo de respuesta del laboratorio clínico al servicio de urgencia del Hospital del Niño Doctor José Renán Esquivel durante el segundo trimestre de 2016

Yaremi del Carmen Juárez Moore - Celia Llanusa Ruiz - Johana Gutiérrez Zehr (42:56)

\section{DISCUSIÓN}

En esta investigación se identificó que la sección que posee el mayor porcentaje de informes de laboratorio clínico fuera de tiempo es hematología general con un 40,62\% seguida de la sección de química general con un 25,14\%. Los turnos con mayores problemas en la respuesta fueron: 7am-3pm y 3pm- 9pm los días de semana, especialmente en el horario de 7am-9:30 am, lo que debe ser materia de evaluación.

Todas las secciones cumplen con tiempos de respuestas globales menores a 1:00:00 hora, pero existen tendencias de tiempos de respuesta prolongados en los horarios matutinos y vespertinos. Si bien es cierto no existen estudios exactamente iguales al realizado, pero podríamos comparar con algunos muy similares con hospitales similares en capacidad, tal es el caso del Hospital Inglesa en Ceuta, en donde los tiempos para el servicio de urgencia fueron: Bioquímica (Química General) 45 minutos; Hematología (45 minutos); urinálisis 30 minutos. En ese estudio, las causas más frecuentes en los atrasos de los tiempos de respuesta encontrados fueron: la demora en la recepción de la muestra, problemas con identificación de la muestra que requiere en todo caso que se aclare antes de poder procesarlo, así como avería de los sistemas informáticos y de los equipos automatizados (Cascante, 2007).

En la bibliografía consultada, no se encuentran trabajos que evalúen exactamente los tiempos de respuestas que hemos valorado y la metodología de estudio utilizada por lo cual sólo podemos comparar parcialmente con los de Belzunegui O, Garcia N, Eguiluz G (1993) y el de Garcia V, Claveria F (1989). En este último la demora de los tiempos de respuesta es mayor en horas de la mañana, afectando el tiempo de respuesta en la sala de urgencias de manera global, colapsando el servicio de urgencia durante las horas de la tarde ya que suman tiempos de espera de un turno y otro.

Como acciones correctivas propuestas dentro del plan de mejoramiento se sugiere a la institución establecer en conjunto con el servicio de urgencia, los tiempos de repuesta adecuados para los distintos análisis, estandarizar de acorde a la demanda y necesidad del servicio, un cuadro básico de urgencia de acuerdo con las estadísticas, de manera que se puedan optimizar los recursos disponibles, indicar al personal de la sala de urgencia que las solicitudes deben llenarse de forma completa, tanto en datos demográficos, como en los casos que se tenga pacientes con diagnósticos previos, ya que esto facilita al tecnólogo médico el proceso, y puede brindar información adicional que aporte al diagnóstico del paciente por parte del clínico.

Atendiendo a estándares de calidad internacionales se deben revisar periódicamente los tiempos de respuestas, de manera tal que se pueda reducir y evitar atrasos no justificados, evaluar la necesidad que pueda existir de reforzar turnos, revisar los procesos o distribución adecuada del 
personal, de manera que se puedan tomar acciones inmediatas para mejorar los tiempos de respuesta, realizar encuestas al personal que participa en los procesos pre analíticos, analíticos y post analíticos, a fin de dar con las posibles causas de errores y atrasos en los tiempos de respuesta, el registro de incidencias y no conformidades puede contribuir a analizar y darle seguimiento adecuado; y en cuanto a los equipos automatizados, se debe revisar y evaluar los tiempos promedios para cada uno de los análisis que realizan, el tiempo que toma la realización de los mantenimientos de los mismos, ya que una correcta escogencia de los equipos (ver pruebas por horas) es primordial para mejorar el proceso analítico.

Por último se sugiere a la institución implementar estudios de TR en el área de microbiología, ya que no fueron contemplados en esta investigación y podrían aportar información relevante, como la obtenida en esta investigación para continuar brindando atención con calidad, bajo la política internacional de seguridad del paciente.

\section{REFERENCIAS BIBLIOGRÁFICAS}

Cascante, T. C. (2007). Análisis de los Tiempos de Respuesta del Laboratorio Clínico para el Servicio de Urgencias del Hospital San Rafael de Alajuela durante los meses de mayo a septiembre del 2007. Costa Rica

García Lario , J. V., \& Boned J. , B. (2012). Control de la calidad interno: una práctica muy actual. educación continuada en el laboratorio clinico.

García Vega, J., \& Claveria Fontan , A. (1989). Tiempo de estancia en un servicio de urgencia hospitalario. Emergencias.

Hospital del Niño Doctor José Renán Esquivel. (2014). Boletín estadístico 2014. Panamá International Organization for Standardization (2012). Disponible en: https://www.iso.org/ home.html. Consulta 24/02/2017.

Moraga Moraga , H., \& Gonzalez Cascante, R. (2011). Satisfacción del usuarioque requiere exámenes urgentes, Costa Rica. Revista Costa Rica Salud Pública.

Pérez-Jáuregui , J., \& Limón-Figueroa, M. (2008). Tiempo de entrega de resultados de laboratorio en el departamento de urgencias: Satisfacci\{on de los médicos? Medigraphic Artemisa en línea. Disponible en: http:/www.medigraphic.com/pdfs/patol/pt-2008/pts081a. pdf Consulta: 19/01/2017 
Shmuklerman, B. J. (2010). Tiempo de respuesta como indicador de calidad en el laboratorio de terapia Intensiva. http:/www.monografias.com/trabajos78/tiempo-respuesta-indicadorcalidad-laboratorio/tiempo-respuesta-indicador-calidad-laboratorio2.shtml. Consultado: $12 / 11 / 2016$

Salinas, M., López-Garrigós, M., Yago, M., Ortuño, M., Díaz, J., Marcaida, G., \& ... Uris, J. (2011). Regional pilot study to evaluate the laboratory turnaround time according to the client source. Revista De Calidad Asistencial: Organo De La Sociedad Española De Calidad Asistencial, 26(2), p.104-110.

White, B. (2002). The impact of ISO 15189 and ISO 9001 quality management systems on reducing errors. Vox Sanguinis, 83 Suppl 117-20

Werfen Company. (2014). Werfen Company. Obtenido de Werfen Company. Didponible en: http://www.systelabsw.com/products/modulab.aspx Consultado: 27/02/2017 